\title{
Effects of UV Intensity, Line-speed, and Light Distribution on Conversion and Surface Elastic Modulus of Roll-to-roll UV-cured Film
}

\author{
Kentaro Taki ${ }^{1 *}$, Shunsuke Kondo ${ }^{2}$, and Hiroshi Ito ${ }^{2}$ \\ ${ }^{1}$ Chemical and Materials Engineering Course, School of Natural Systems, College of Science and \\ Engineering, Kanazawa University, Kakumacho, Kanazawa, Ishikawa, 920-1192, Japan \\ ${ }^{2}$ Department of Polymer Science and Engineering, Yamagata University, 4-3-16, Jonan, \\ Yonezawa, Yamagata, 992-8510, Japan \\ E-mail:taki@se.kanazawa-u.ac.jp
}

\begin{abstract}
Roll-to-roll UV nanoimprint lithography (R2RUVNIL) is a promising technology for fabricating nanostructures on flexible films, but its application requires finding feasible operating conditions to peel-off the UV resin from the mold. Furthermore, to achieve the required nanostructure, conversion of the UV resin needs to be precisely optimized in terms of the UV intensity and line speed. In this study, (1,6-bis(acryloyloxy)hexane) resin was cured by roll-to-roll UV-curing while monitoring its conversion and surface elasticity by Fourier transform infrared spectroscopy and a nano indenter, respectively. It was found that the conversion increases by up to $85 \%$ with a decrease in line speed and increase in UV intensity. The effect of varying the distribution of UV light on the roller mold was also investigated, which revealed that a sharp distribution of UV light is effective in increasing the surface elastic modulus from 0.2 to $1.0 \mathrm{GPa}$ at 0.6 conversion.
\end{abstract}

Keywords: roll-to-roll, UV-curable resin, conversion, elasticity

\section{Introduction}

Roll-to-roll (R2R) UV nanoimprinting is a fast and precise production process that can be used to create large $(10-1000 \mathrm{~nm}$ sized) nanostructured patterns of cross-linked polymers on a plastic substrate film. This is achieved by applying a very thin $(<20 \mu \mathrm{m})$ coating of UV-curable resin onto a plastic film through a slot die coater, which is then pressed into a nanomold. The low viscosity of the resin allows it to permeate and create a conformal coating; and at the same time, it is photopolymerized by UV light irradiation. The cured coating is then stripped by rotating the nanomold and translating the film.

There have been numerous attempts to develop a more precise $R 2 R$ process [1-6] so as to take greater advantage of the unique structural colors $[2,3]$ and dewetting properties [7] afforded by nanostructured surfaces.
Although continual advancement in mold fabrication technology is certain to increase the number of commercial applications for continuous R2R imprinting, the high cost of nanostructured molds for UV nanoimprinting remains an issue. Indeed, the only way at present to achieve the desired characteristics in a nanoimprinted product is to modify a nanostructured mold through trial-and-error, which greatly increases the total cost.

Acrylic resins, which are sensitive to oxygen, are currently the most widely used resins. These are produced by fast radical polymerization, and so are suitable for high throughput applications [4,8]. However, partially polymerized resins can cake onto the mold, making it necessary to clean or discard it. This not only reduces throughput, but also increases the cost [4].

While the cause-and-effect relationship
Received November 2, 2016

Accepted November 26, 2016 
between the operating conditions and nanostructures produced by the roll-to-roll UV nanoimprint process (R2RUVNIL) have been well studied, little attention has been given to how UV-curable resins are photo-polymerized. What is known is that UV resins dramatically change from a purely viscous liquid to an elastic solid, and that this change in viscoelasticity can cause catastrophic damage to a nanomold.

The optimal degree of conversion in a UV curable resin is shown schematically in Fig. 1 (modified from ref [1]). This shows that if the UV resin does not fully cure, then residual resin will be left in the nanomold. An accumulation of this un-cured resin in the nanomold will decrease the possible printing cycle; and if the nanomold is exposed to UV light without a substrate film, then the uncured resin can readily cure in the nanomold and be hard to remove. This ultimately makes replication of the nanostructure on the nanomold impossible. On the other hand, if the UV resin is cured too much, then it will bond strongly to the nanomold and prove difficult to detach. In this case, the nanomold can no longer be used.

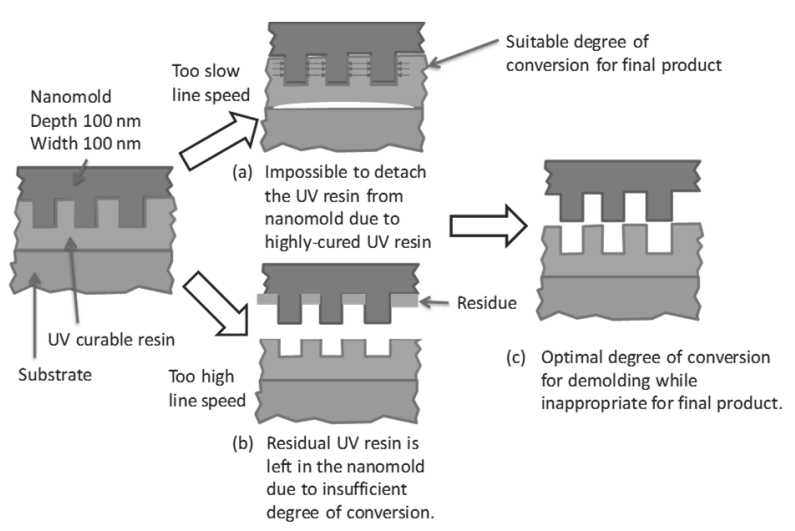

Fig. 1. Concept for optimal degree of conversion in UV curable resin during modified R2R nanoimprinting process [1].

Figure 2 illustrates a concept for the optimal degree of conversion based on the force of adhesion between the nanomold and UV resin $\left(F_{M}\right)$, and between the UV resin and substrate $\left(F_{S}\right)$. For the UV-resin to detach from the nanomold it is essential that $F_{M}$ be lower than $\mathrm{F}_{\mathrm{S}}$, or else the nanomold will become caked with UV-resin. Figure 2 (b) shows a speculative relationship between $F_{M}$ and $F_{S}$ with increasing conversion of the UV resin, which can be divided into three distinct areas. The area of "too low conversion" indicates that the UV-resin is not sufficiently cured to replicate the nano structure of the nanomold. The area close to the intersection of the $F_{M}$ and $F_{S}$ curves is the feasible area, in which $F_{M}<F_{S}$ and the UV resin is sufficiently cured. When the conversion of the UV-resin exceeds this point, de-molding (detachment) becomes infeasible. To the best of our knowledge, no on-line measurement of the adhesion force has ever been achieved, thus making it very challenging to define the feasible area. There is therefore a need for suitable on-line measurement technology to ensure stable commercial fabrication.

During industrial operation of R2RUVNIL the nanomold is coated with a release agent [5], which causes the FM curve to shift to the bottom of the graph in Fig. 2 (b). This prevents UV resin being caked into the nanomold, but the gradual loss of the release agent that occurs during the process implies that it needs to be constantly reapplied to maintain the surface condition of the nanomold.

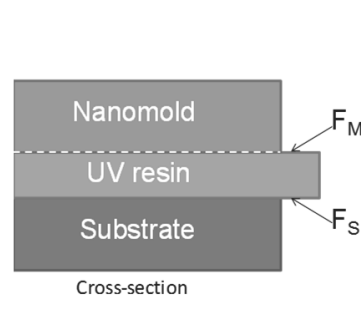

(a)

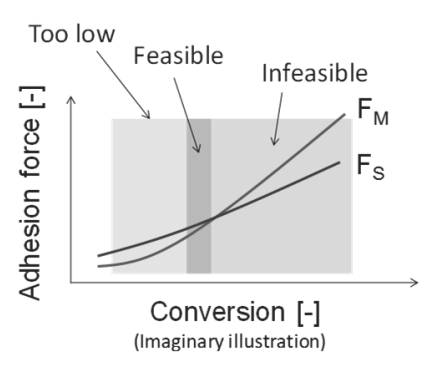

(b)
Fig. 2. (a) Schematic of adhesion forces for nanomold-UV resin $\left(F_{M}\right)$ and $U V$ resin-substrate $\left(F_{S}\right)$. (b) Speculated relationship between $F_{M}, F s$, and critical point of good de-molding (detachment).

A special formulation consisting of a mix of several kinds of UV-curable monomers, photoinitiators and additives has been used with R2RUVNIL, but this makes kinetic and analytical measurements difficult to perform either in situ or ex situ. However, the degree of conversion and related physical properties (e.g., strength of adhesion, viscoelasticity, and surface tension) are very important to understanding the process. In this preliminary study, a single-component, di-functional UVcurable resin with a clearly defined chemical structure and relatively high purity was used to 
measure the degree of conversion and change in surface elastic modulus using Fourier transform infrared spectroscopy (FT-IR) and a nano indenter. Through a series of experiments, the effect of changing the line (web) speed, UV intensity, and UV light distribution were assessed and are herein discussed.

\section{Experimental}

\subsection{Materials}

A di-functional, UV-curable resin (1,6bis(acryloyloxy)hexane stabilized with MEHQ, B2936) was supplied by Tokyo Chemical Industry, Japan. The photoinitiator used was Irgacure 184, which was supplied by BASF Japan. All chemicals were used without further purification and were mixed to a ratio of 99:1 UV curable resin to photoinitiator by weight. The substrate was a $100 \mu \mathrm{m}$-thick polyethylene terephthalate (PET) film.

\subsection{R2R UV Nanoimprint Process}

The R2R UV nanoimprint device used in this study was $2.5 \mathrm{~m}$ wide and $1.5 \mathrm{~m}$ high with seven rollers in total and was manufactured by TOSHIBA machinery, Japan. A photo of this device is provided in Fig. 3, and a schematic diagram of the process is shown in Fig. 4. During operation, a web of film was installed at the bottom of the slot die coater, and was then drawn out and attached onto the roller directly in front of the slot die coater (Fig. 5). The UV-curable resin was then pumped from a reserve tank through the slit of the slot die coater and onto the film. The gap between the coater and film was adjusted by a micrometer connected to the roller.

Once the UV-curable resin was applied, the film was drawn onto the $200 \mathrm{~mm}$-diameter nanomold roller shown in Fig. 6 and pressed against it with a silicone roller. The UV light emitted by a high-pressure mercury lamp located below the roller was used to immediately cure (photopolymerize) the resin through the substrate film. The highest UV intensity was typically in the order of 160 $\mathrm{mW} \cdot \mathrm{cm}^{-2}$. Once cured, the film was detached from the nanomold roller onto a film winder.

As the nanomold roller was exposed to the UV light without any form of lamp shade, a 10 mm-wide slit was installed to confine the UV light and examine the effect of varying its distribution. A UV scale ${ }^{\circledR}$ (Fuji Film, Japan), which changed color in response to UV intensity multiplied by the exposure time, was attached to the nanomold and observed at $10 \mathrm{~s}$

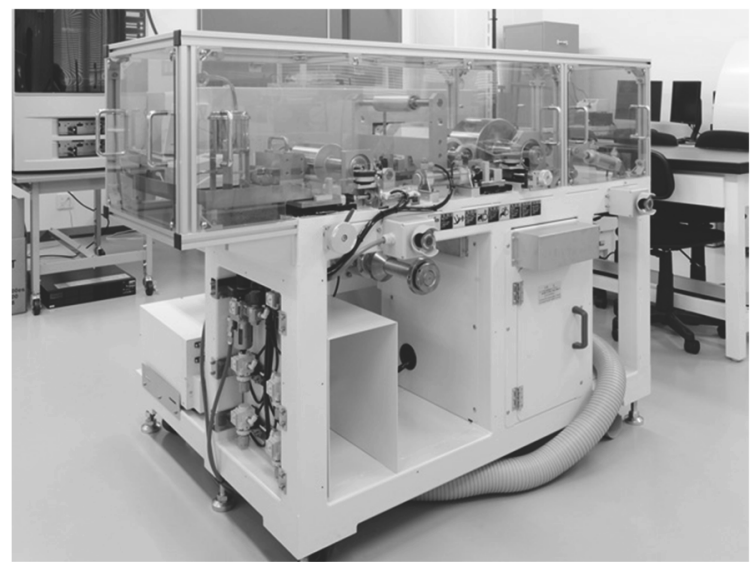

Fig. 3. Roll-to-roll UV nanoimprinter.
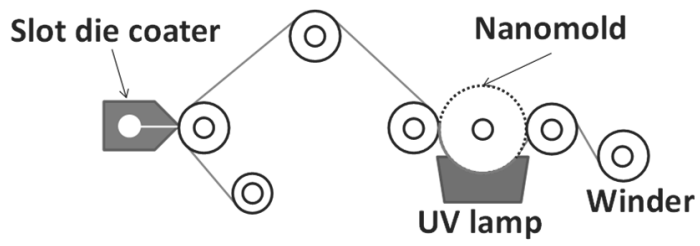

Fig. 4. Schematic diagram of roll-to-roll nanoimprint process.

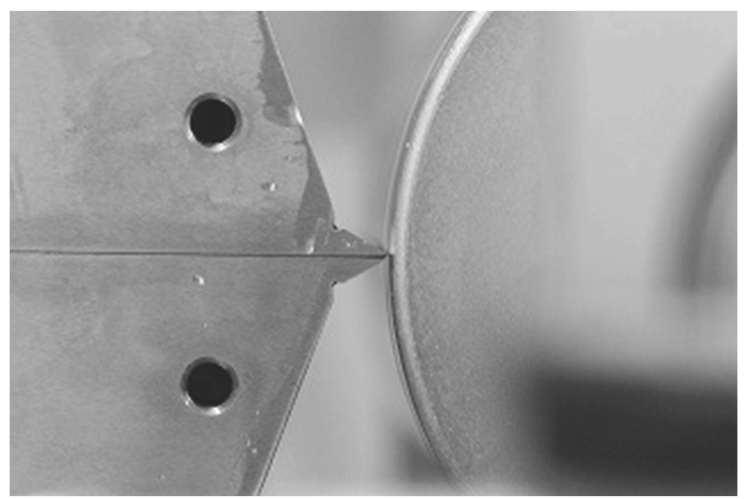

Fig. 5. Picture of slot die coater, UV curable resin, film, and roller. The film was drawn from bottom to top.

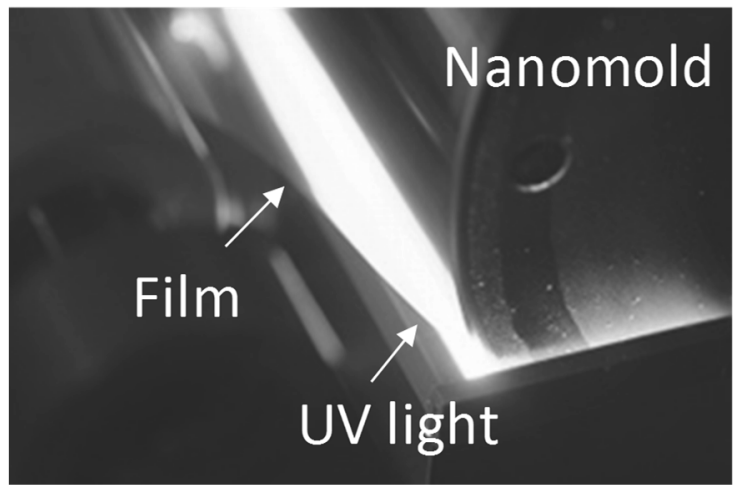

Fig. 6. UV irradiation during nanoimprint process. 
intervals. As shown in Fig. 7, the slit produced a sharper and narrower UV distribution, while still maintaining the same maximum UV intensity.

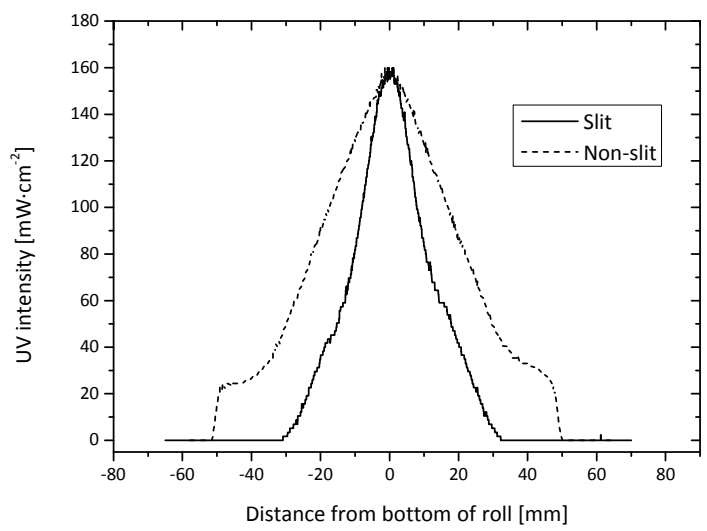

Fig. 7. Distribution of UV light with and without slit on UV lamp.

\subsection{Conversion of UV-cured resin}

The UV-curable resin has two acrylate groups per molecule, and exhibits a characteristic peak at 812 $\mathrm{cm}^{-1}$. The absorbance at $812 \mathrm{~cm}^{-1}$ was therefore measured by FT-IR (VERTEX 70, Bruker-Optics, Germany) and an optical transmittance bench [9] Conversion of the acrylate group was considered proportional to the absorbance when normalized by the film thickness. The conversion of the acrylate group was defined according to the Lambert-Beer law as follows:

$$
x=1-\frac{A_{812}(t) / L(t)}{A_{812}(0) / L(0)}
$$

where $A_{812}(0)$ and $A_{812}(t)$ are the absorbance of the UV-cured resin at $812 \mathrm{~cm}^{-1}$ before and after UV irradiation, respectively. The terms $L(t)$ and $L(0)$ represent the thicknesses of the cured film and a 10 $\mu \mathrm{m}$ spacer, respectively. The $A_{812}(0)$ absorbance was measured by sandwiching the resin between two polished $\mathrm{KBr}$ plates using the $10 \mu \mathrm{m}$ spacer to maintain a consistent gap.

The thickness of the UV-cured resin film on the substrate was varied by changing the line speed and resin flow rate. As the absorbance is the product of molar extinction coefficient, thickness and the molar concentration of the acrylate group, the measured absorbance was divided by the film thickness to remove the effect of the latter. The film thickness was measured with a micrometer (Mitutoyo, Japan), which had a resolution of $1 \mu \mathrm{m}$.
2.4. Surface elastic modulus of UV-cured film

The surface elastic modulus of the UV-cured film was measured with a Berkovich-type nano indenter (G200, MTS). As the relationship between the surface elastic modulus and indentation depth became flat at more than $500 \mathrm{~nm}$, and indentation depth of 2,000 $\mathrm{nm}$ was used. The surface elastic modulus of each point was calculated by averaging the data points between 500 and 1,000 $\mathrm{nm}$. The average and standard distribution were then obtained from nine points $(100 \times 100 \mu \mathrm{m})$ on the UV-cured film.

\section{Results and Discussion}

The FT-IR spectra of both the UV-curable resin and UV-cured resin film are shown in Fig. 8. The UV-cured film was peeled from the substrate by attaching adhesive tape, and was then mounted on the sample stage using tweezers. Note that there are three distinct peaks consist with carbon-carbon double bonds at $1,635,1,408$, and $812 \mathrm{~cm}^{-1}$. The peak at $812 \mathrm{~cm}^{-1}$, as indicated by an arrow, was selected for evaluating the conversion of the UV-curable resin. As shown in the figure, the height of this peak dramatically decreased upon photopolymerization.

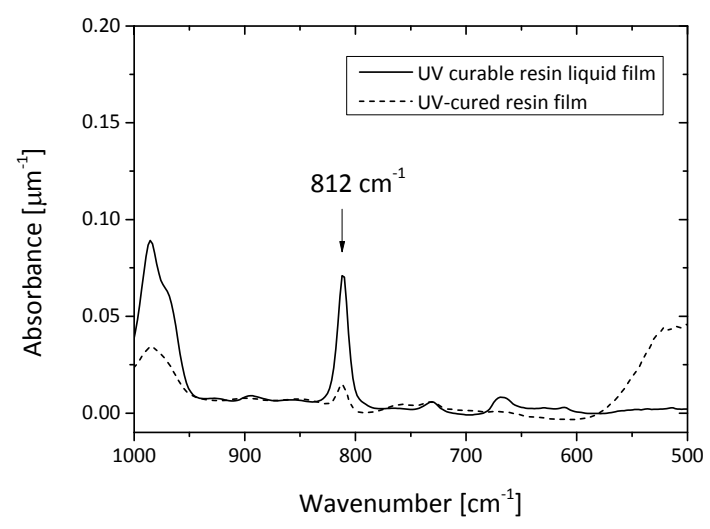

Fig. 8. FT-IR spectrum of UV-curable resin (red) and UV-cured film (black).

Figure 9 shows the effects of line speed and UV intensity on conversion. From this we observe that the conversion decreased with increasing line speed, which is inversely proportional to the UV exposure time. In other words, it is the short exposure time that is created by an increase in line speed that causes a low conversion of the UV-curable resin. An 
increase in the UV intensity from $70 \%$ to $80 \%$ increased the conversion at every line speed, as the higher UV intensity is more effective in dissociating the photoinitiator. This causes an increase in the number of initiation and propagation reactions of photopolymerization, which increases the rate of conversion.

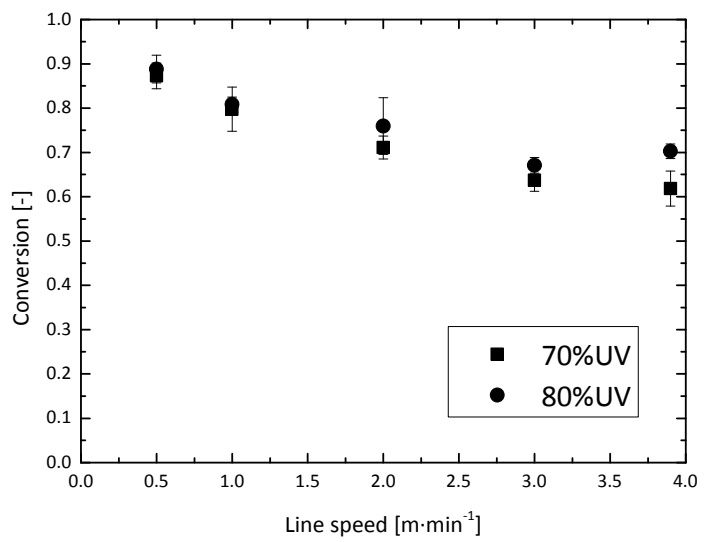

Fig. 9. Effect of line speed and UV intensity on conversion of acrylate group in resin films cured with UV intensities of $80 \%$ and $70 \%$.

The effect of line speed on the surface elastic modulus at a UV intensity of $80 \%$ without a slit is shown in Fig. 10. This reveals that the surface elastic modulus decreases with increasing line speed, which can be attributed to the decrease in the conversion of the UVcurable resin. Figure 10 also shows the effect of UV intensity; which as expected, reveals that a decrease in UV intensity from 80 to $60 \%$ causes a decrease in the elastic modulus at any given line speed. This is again related to a decrease in the conversion of the UV-curable resin.

Figure 11 shows the relationship between the surface elastic modulus and conversion of the UV-curable resin. Although the data points were scattered when no slit was used, the overall trend indicates that the surface elastic modulus changes with conversion. When a slit was used, the increased sharpness of the UV light indicates that the surface elastic modulus was higher for a given conversion due to the light being confined to the nanomold. The exact reason why a high-modulus UV-cured film can be obtained by installing a slit is still under consideration, but is believed to be related to a difference in the network structure of the UV-cured resin.

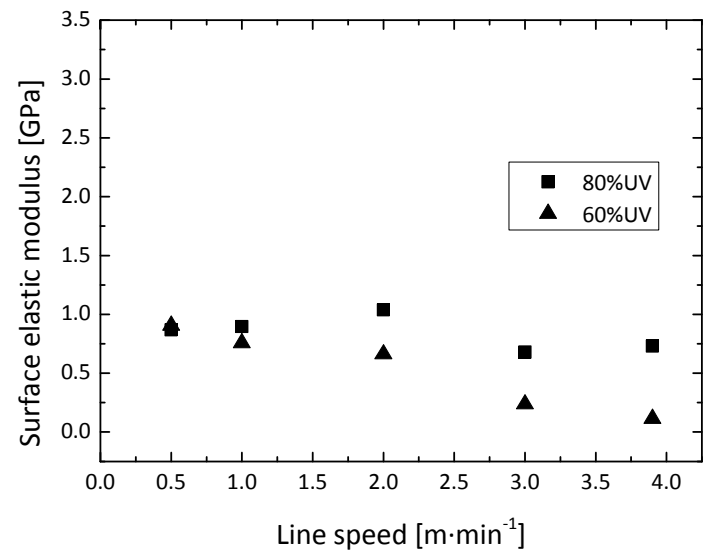

Fig. 10. Effect of line speed and UV intensity on surface elastic modulus (no slit).

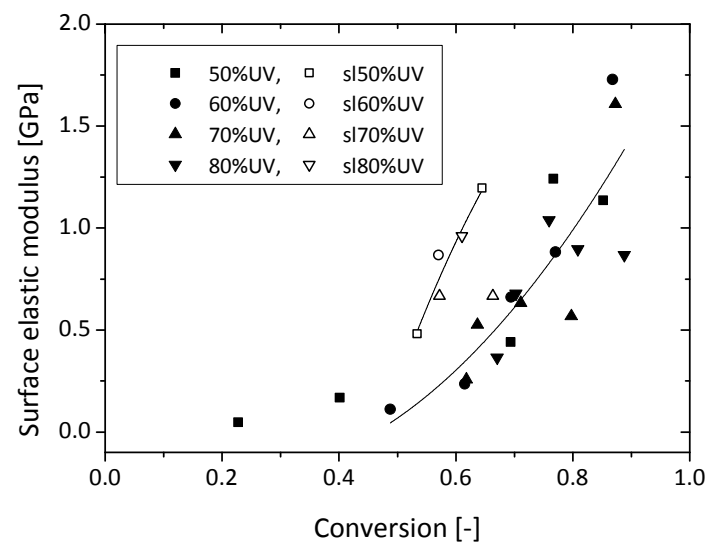

Fig. 11. Relationship between surface elastic modulus and conversion of UV-curable resin. The "sl" indicates the result of surface elastic modulus with the slit.

Figure 12 shows a preliminary illustration of the network structures that are believed to be produced with and without a slit. Here, the broad light-distribution produced without a slit causes the photo initiator to undergo a gradual initiation reaction in the UV-curable resin, which results in a broad chain length

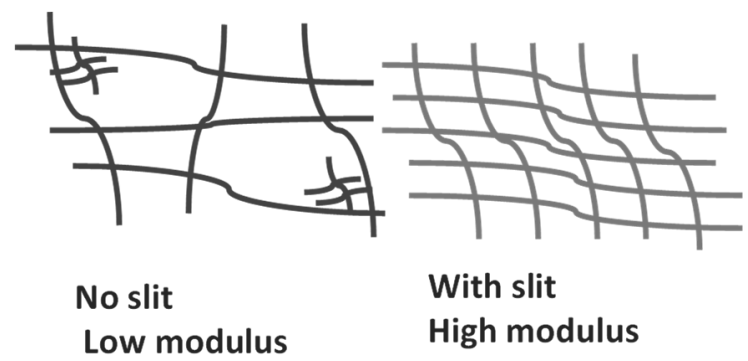

Fig. 12. Network structure formed in UV-cured films with and without a slit. 
distribution and uneven network structure. A sharper distribution of UV light, however, causes the initiation reaction to occur simultaneously, producing a more uniform network structure with a higher elastic modulus.

\section{Conclusion}

In this preliminary study of R2R UV nanoimprinting, a UV-curable resin was coated onto a substrate film and cured on the roller by UV irradiation. Once removed from the substrate, the UV-cured film was subjected to FT-IR measurement, which demonstrated that the thickness-normalized absorbance increases with increasing line speed and decreasing UV intensity. This indicates that the conversion of the UV-curable resin varies with UV intensity and line speed.

A slow line speed and high UV intensity were found to increase the surface elastic modulus of the UV-cured film due to an increase in the degree of conversion. Varying the UV light distribution on the nanomold by installing a slit on the UV lamp also increased the surface elastic modulus, which is believed to be the result of a more uniform network structure.

\section{References}

1. H. Schift, J. Vac. Sci. Tech. B, 26 (2008), 458.

2. S. H. Ahn, L. J. Guo, Adv. Mater, 20 (2008) 2044.

3. S. H. Ahn, L. J. Guo, ACS Nano, 3 (2009) 2304.

4. J. J. Dumond, H. Y. Low, J. Vac. Sci. Technol. B, 30 (2012), 010801-1.

5. H. Yoshikawa, J. Taniguchi, G. Tazaki, T. Zento, Microelectron. Eng., 112 (2013) 273.

6. Y. Suto, T. Mizawa, Y. Mizukami, T. Takahashi, J. Photopolym. Sci. Tech., 26 (2013) 125.

7. A. Pozzato, S. D. Zilio, G. Fois, D. Vendramin, G. Mistura, M. Belotti, Y. Chen, M. Natali, Microelectron. Eng., 83 (2006) 884.

8. M. D. Dickey, R. L. Burns, E. K. Kim, S. C. Johnson, N. A. Stacey, C. G. Willson, AIChE J., 51 (2005) 2547.

9. K. Taki, Y. Watanabe, H. Ito, M. Ohshima, Macromolecules, 47 (2014) 1906. 